\title{
Numerical simulation of tonal and broadband hydrodynamic noises of non- cavitating underwater propeller
}

\author{
B. Mousavi, Ph. D. student \\ A. Rahrovi, Ph. D. student. \\ S. Kheradmand, Assis. Prof. ${ }^{*}$ \\ ${ }^{*}$ Malek-Ashtar University of Technology, Shahin-shahr, Iran
}

\begin{abstract}
The objective of the study was to carry out numerical simulation of the hydrodynamic noise generated by the flow around a non-cavitating underwater propeller. To achieve this goal, hydrodynamic simulation of flow around the propeller was initially done. The unsteady 3-D flow was modelled numerically along with the LES turbulence model. The hydrodynamic parameters calculated for different advance coefficients are visibly in line with the previous experimental works. The turbulent quantities of the hydrodynamic study and the FWH model were used to find spectral distributions of flow noise for different advance coefficients. The results of the acoustic investigation were compared against other numerical results. An array of 100 hydrophones was used to find the directional distribution of the noise around the propeller. The obtained results indicate that, for different advance coefficients, the highest intensity of the noise recorded by different receivers around the propeller occurs in BPF. Furthermore, it has been found that the noise is directionally as well as intensively distributed around the propeller. Noise distributions of noise are presented and discussed for different regimes of propeller rotation. The analysis of the expanded spectrum (broadband analysis) of noise on the propellers has also been done and the contribution of all parts of the propeller to hydrodynamic noise generation are presented.

Keywords: Numerical Simulation,tonal, broadband, Hydrodynamic Noise, Propeller
\end{abstract}

\section{INTRODUCTION}

The noise generated by the propeller of a submarine is the most principal factor in its recognition by enemy's sonar contacts. The noise also pollutes the subsea environment. Hence, it seems vital to measure the noise and lower its intensity. The noise produced by the propeller can be divided into that of cavitational and non-cavitational nature. Although most propellers experience cavitation and generate enormous noise due to the destruction of bubbles, in deep see conditions propellers are still noisy without cavitation.

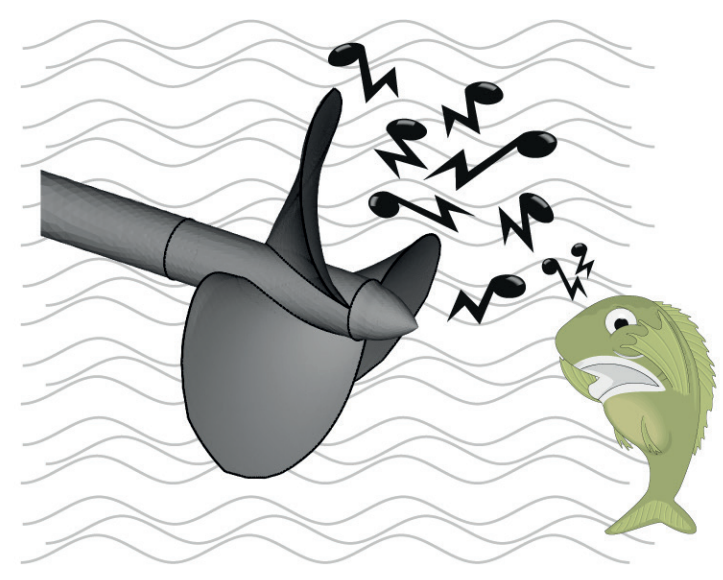

Fig. 1. Underwater propeller DTMB 4119
The hydrodynamic analysis of the flow around the propeller is the prerequisite of its acoustic analysis. Propellers rotate in a highly turbulent wake behind vessels, therefore the flow pattern around them is truly complicated and hard to resolve. It seems necessary to precisely simulate the turbulent quantities. In the present study, the flow around the propellers is analysed both numerically and experimentally. Many researchers measured experimentally propellers performance [1-4]. Unfortunately, these methods require complex laboratory equipment and are very expensive, especially when fluctuating quantities are to be measured. On the other hand, numerical simulations (making use of both inviscid [5-10] and viscous flow methods [11-15]) are also widely represented in the literature.

Turbulence modelling is the most challenging problem of propeller hydrodynamic studies. Many researchers use RANS equations including $0,1,2$ and multi-equation methods to resolve the closure problem [11-15]. In a pure hydrodynamic study oriented on determining certain integral quantities including $\mathrm{K}_{\mathrm{t}}$ or $\mathrm{K}_{\mathrm{Q}}$, the given methods lead to acceptable results. However, in the noise studies where temporal and space distributions of fluctuating quantities, such as pressure for instance, should be found, more accurate models are required. Here, DNS [16], DES [17] and LES [18] are the candidates to model the turbulence. LES is more commonly used to setup hydrodynamic parameters for acoustic investigations, because of its precision and moderate computational cost [19-22]. 
The noise detected in the fluid can be generated by either a vibrating structure or fluid fluctuations. Although the structural noise is very important and is studied by many researchers [23-25], in the present work the vibration of the propeller structure is not considered and only the flow noise is studied.

Sound propagation is related to the media in which the noise is propagated. Although the subject studied by most flow noise researches falls into the category of aero-acoustic noise (in gas media) [26], the hydrodynamic noise has also been the object of interest of some researches [27]. Regardless of the media, basically there are two kinds of methods to model the flow noise numerically, which are the direct methods [28] and those based on the acoustic analogy [29]. Beside the acoustic analogy, some methods, such as the Linearised Euler Equation [30-32], the Acoustic Perturbation Equation [33] and the Linearised Perturbed Compressible Equation make use of perturbation quantities [34].

In the present study, the flow-noise of a rotating submerged propeller is predicted by a 3D numerical simulation. For the near-field, the Navier-Stocks Equations are used for modelling the flow and the LES model to model the turbulence, while for the far-field acoustic, the FWH model is applied. Equations are presented in Section 2, and the obtained results including the hydrodynamic and tonal noise results are presented in Section 3. Broadband noise results are presented in Section 4 and, finally, in Section 5 concluding remarks are depicted.

\section{EQUATIONS AND NUMERICAL SOLUTION}

In the hydrodynamic analysis of the flow, the flow field can be predicted by solving the continuity and momentum equations (Equations 1 \& 2) [18].

$$
\begin{gathered}
\frac{\partial \rho}{\partial t}+\frac{\partial}{\partial x_{i}}\left(\rho u_{i}\right)=0 \\
\frac{\partial}{\partial t}\left(\rho u_{i}\right)+\frac{\partial}{\partial x_{k}}\left(\rho u_{k} u_{i}\right)=\rho g_{i}-\frac{\partial P}{\partial x_{i}}+\frac{\partial \tau_{i j}}{\partial x_{j}}
\end{gathered}
$$

where ui are velocity components of water, $\rho$ is density, $\mathrm{P}$ is pressure, and $\tau$ is the shear stress tensor.

For the acoustic analysis of the propeller the integral equation FWH (Equation 3) is solved to find the far-field sound of the propeller [35].

$$
\begin{aligned}
& \left.\frac{1}{a_{0}^{2}} \frac{\partial^{2} p^{\prime}}{\partial t^{2}}-\nabla^{2} p^{\prime}=\frac{\partial^{2}}{\partial x_{i} \partial x_{J}}\left\{T_{i j} H(f)\right\}-\frac{\partial}{\partial x_{i}}\left\{P_{i j}+\rho u_{i}\left(u_{n}-v_{n}\right)\right] \delta(f)\right\} \\
& +\frac{\partial}{\partial t}\left\{\left[\rho_{0} v_{n}+\rho\left(u_{n}-v_{n}\right)\right] \delta(f)\right\}
\end{aligned}
$$

In Equation 3 p' is the pressure fluctuation and $\mathrm{T}_{\mathrm{ij}}$ is the Lighthill stress tensor. The following equation is referred to as the Lighthill's equation in which the first right-side term stands for the generation source of the monopole noise, the second term represents the dipole noise, and the third term accounts for the quadrupole noise[35].

$$
\frac{\partial^{2} \rho}{\partial t^{2}}-c^{2} \frac{\partial^{2} \rho}{\partial x_{i}^{2}}=\frac{\partial q}{\partial t}-g_{i} \frac{\partial \rho}{\partial x_{i}}+\frac{\partial f_{i}}{\partial x_{i}}-\frac{\partial^{2} \tau_{i j}}{\partial x_{i} \partial x_{j}}
$$

In this study, the noise caused by the rotating propeller forms the monopole thickness noise. The noise of the pressure level and propeller suction shapes the dipole loading noise. As the Mach number of the propeller rotation is small, the volume noise is ignored. Using the Lighthill analogy, Proudmam proposed the following formula to compute the acoustic power of the turbulent flow, regardless of the average flow [36].

$$
P_{A}=\alpha \rho_{0}\left(\frac{u^{3}}{l}\right) \frac{u^{5}}{a_{0}^{5}}
$$

In the above equation, $\mathrm{u}$ and $\mathrm{l}$ are the turbulence velocity and the length scale, respectively, while $\mathrm{a}_{0}$ and $\alpha$ are the sound speed and a coefficient.

For the simulation of the broadband noise, the acoustic power on the propeller surface $\left(\mathrm{L}_{\mathrm{p}}\right)$ is derived from Equation 6 .

$$
L_{P}=101 \log \left(\frac{P_{A}}{P_{r e f}}\right)
$$

In the above equation, $\mathrm{P}_{\text {ref }}$ is the reference acoustic power and equals $10-12 \mathrm{~W} / \mathrm{m}^{3}$.

The studied propeller is a real size DTMB 4119 propeller. All flow simulations are done using the OpenFOAM software and acoustic post processing of the data are performed with a homemade code for noise calculations. Figure 2 (on the right) shows the cylinder-shaped computational domain around the propeller. As shown in the figure, a small cylinder with the length of $0.385 \mathrm{D}$ and diagonal of $1.1 \mathrm{D}$ is placed around the propeller. The cylinder is used to revolve the propeller in the rotating reference frame. Figure 5(right) clarifies the dimensions of the solution (see the table for boundary conditions of the issue). The open water condition is applied to the inlet. To employ the LES model of turbulence for the acoustic analysis, the study employed an extensively fine grid (total number of 2750000 cells) upon and around the propeller, in particular where the flow vortex is generated (figure 2 left). The sliding mesh technique is used to model the rotation of the propeller. The small cylinder in figure 2 around the propeller rotates at the propeller speed.
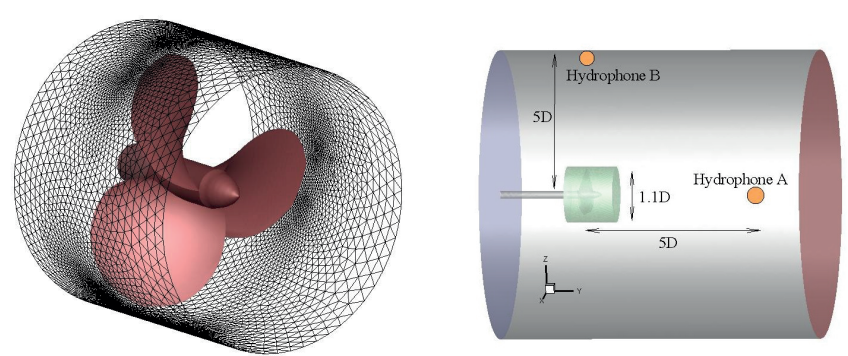

Fig. 2. Computational domain (right) and sliding portion of the domain with unstructured grid (left) 


\section{HYDRODYNAMIC FINDINGS}

Figure 3 shows the graphs comparing the hydrodynamic coefficients as a function of the advance coefficients obtained in the present study and recorded as the laboratory findings [37]. The results are visibly in line with each other. As the graph indicates, for all advance coefficients the thrust and moment coefficients are close to each other and their difference is less than $9 \%$. The value of the advance coefficients was derived from Equation 7.

$$
J=\frac{V_{s}}{N_{p} D_{p}}
$$

where $J$ is the advance coefficient, Vs is the advance velocity, $\mathrm{Np}$ and $\mathrm{Dp}$ are the angular velocity and the diameter of the propeller.

Figures $4 \& 5$ show the pressure coefficients of the propeller blade as functions of $\mathrm{d}_{\mathrm{le}}$ for two different value of $\mathrm{d}_{\mathrm{r}}(0.5$ and 0.7 ) for $J=0.83$. Here $d r$ is defined as $d_{r}=r / R$ in which $r$ is the radial distance from the propeller axis and $\mathrm{R}$ is the propeller radius. The $\mathrm{d}_{\mathrm{le}}$ represents the spatial distance from the propeller blade leading edge, non-dimensionalised by the propeller blade chord. The pressure coefficient value at this section was derived from Equation 8.

$$
C_{p}=\frac{2 p-2 p_{0}}{\rho V_{s}^{2}\left[1+\left(\frac{\pi d_{r}}{J}\right)^{2}\right]}
$$

In the above equation, $C_{p}$ is the pressure coefficient; $p_{0}$ is the reference pressure and $\mathrm{V}_{s}$ is the advance velocity of the propeller. The numerical results of the present study were compared with those obtained by Pan et al. using the panel method, where the fluid was supposed to be inviscid [37]. The existing difference between these two cases is due to the fact that the boundary layer was not modelled in the panel method.

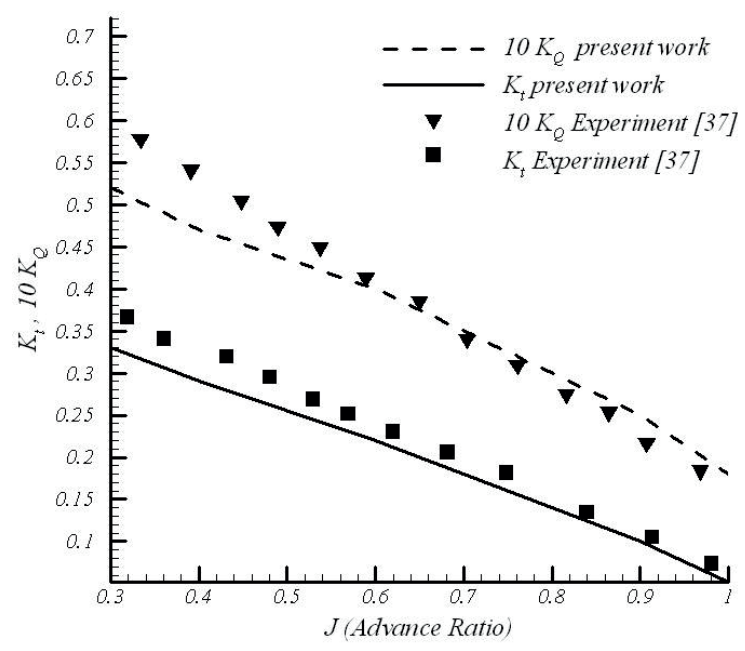

Fig. 3. Hydrodynamic performance of propeller in open water conditions

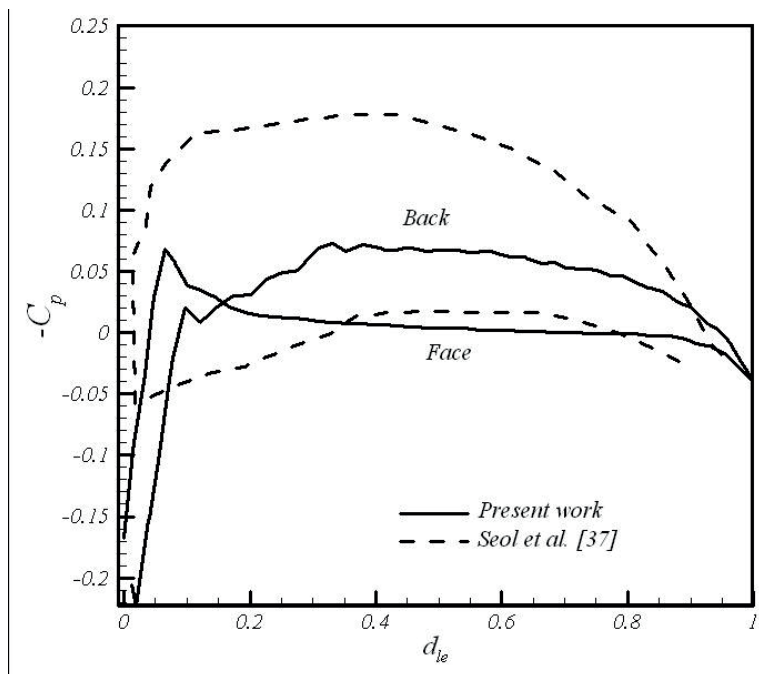

Fig. 4. Pressure coefficients for $d_{r}=0.5$

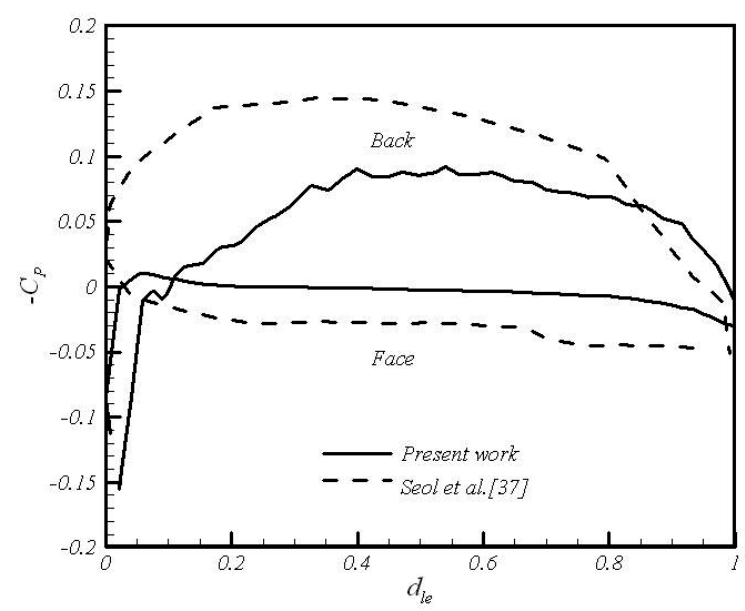

Fig. 5. Pressure coefficients for $d_{r}=0.7$

Figure 6 shows two views of axial distribution of water velocity, where the propeller is rotating with a constant angular velocity of $120 \mathrm{rpm}$. The figure shows the flow pattern in which every blade tip generates a high speed backward flow (regions marked red). At the same time a forward flow with lower axial velocity is also generated. These two reverse flows provoke a vertical flow around the blade tip. As the numerical value of the axial velocity indicates, the rotating direction of the eddy is clockwise.
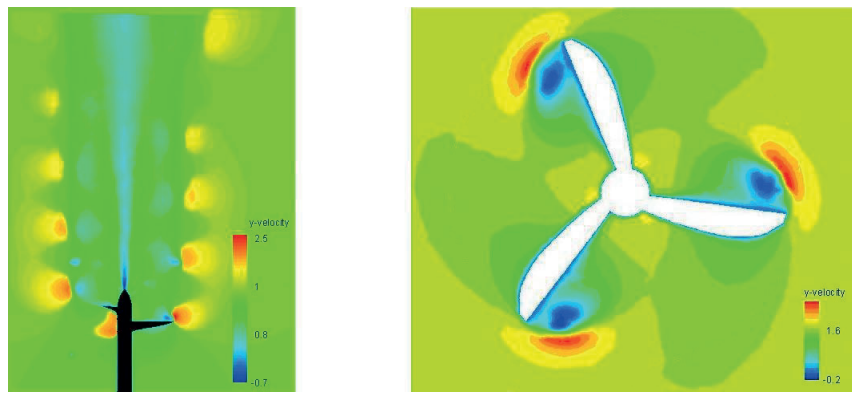

Fig. 6. Axial velocity distribution around propeller in horizontal plane (left) and propeller plane (right) 
Figure 7 shows the planes of the same velocity around the propeller, demonstrating the creation of the radial and tangential flow and the velocity increase from the pure input axial velocity of $1.6 \mathrm{~m} / \mathrm{s}$ to $1.65 \mathrm{~m} / \mathrm{s}$.

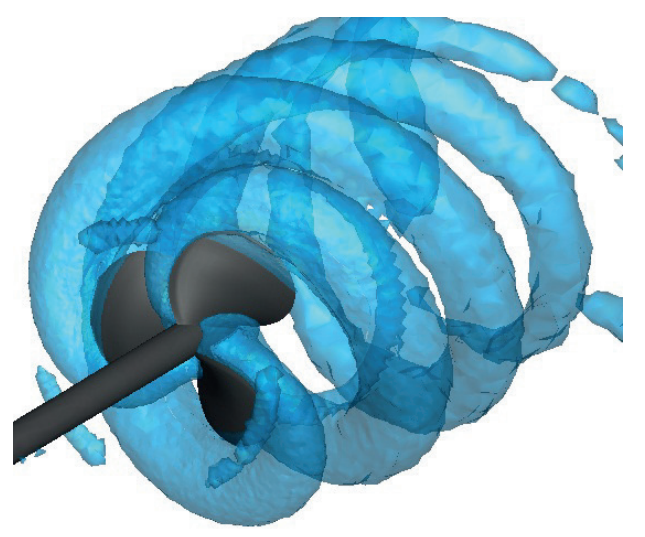

Fig. 7. Velocity isosurfaces, magnitude $1.6 \mathrm{~m} / \mathrm{s}$

Figure 8 shows vorticity contours in two perpendicular planes. As indicated in the figure, blades tips play the main role in vorticity generation. On the other hand it is evident from Figure 8 (left) that the propeller nose is also a source of vorticity. In this case, i.e. for the angular velocity of propeller equal to $120 \mathrm{rpm}$, the magnitude of the tip vorticity is higher than that generated by the propeller nose. The regions of vorticity generation are expected to be an active source of flow noise.
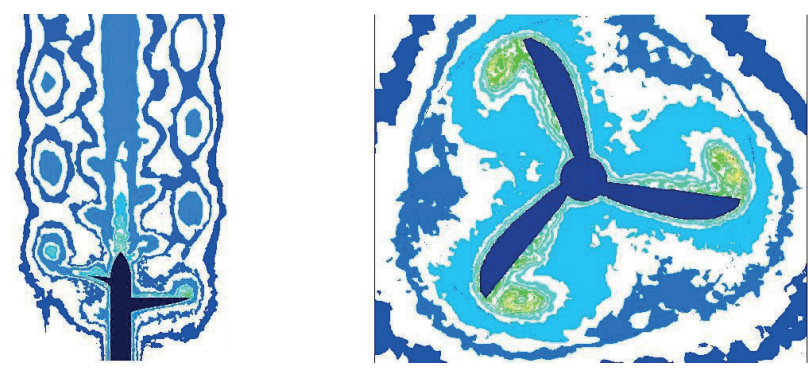

Fig. 8. Vorticity distribution around propeller in horizontal plane (left) and propeller plane (right)

\section{ACOUSTIC FINDINGS}

In this section, the level of the noise generated by the propeller DTMB4119 is presented [38]. The water density and the speed of the sound were assumed as equal to $1026 \mathrm{~kg} / \mathrm{m}^{3}$ and $1500 \mathrm{~m} / \mathrm{s}$, respectively. The level of sound was computed using Equation 9.

$$
S P L=20 \times \log \frac{P_{a c o u s t i c}}{P_{r e f}}
$$

In Equation 9, SPL stands for the decibel based Sound Pressure Level. The level of the reference pressure equals $1 \times 10^{-6}$ pa and $\mathrm{P}_{\text {acoustic }}$ is the acoustic pressure.
Figures 9 \& 10 show noise frequency spectra of two different receivers, one situated upon the hub axis in front of the propeller, at a distance of 10 times of the propeller radius (receiver A Figure 2), and the other located on the propeller rotation plane, above the propeller, also at a distance of 10 times of the propeller radius (receiver B Figure 2). The advance velocity was equal to $1 / 6 \mathrm{~m} / \mathrm{s}$ and the rotational speed was equal to $120 \mathrm{rpm}$. Figures 9 \& 10 evidently reveals substantial compatibility of the findings of the present study with the numerical findings of the study by Seol et al [38]. However, in receiver $\mathrm{B}$, from $100 \mathrm{~Hz}$ on, the difference of $25 \%$ can be observed in the results. This difference may be due to different methods used in two studies, i.e., the present study made use of the CFD method, while Seol et al., used the panel method.

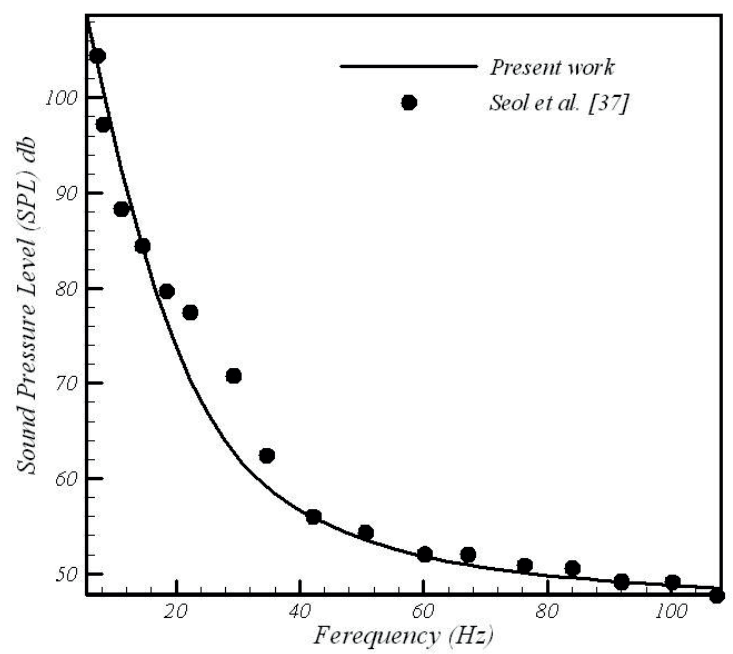

Fig. 9. Noise spectrum in receiver A

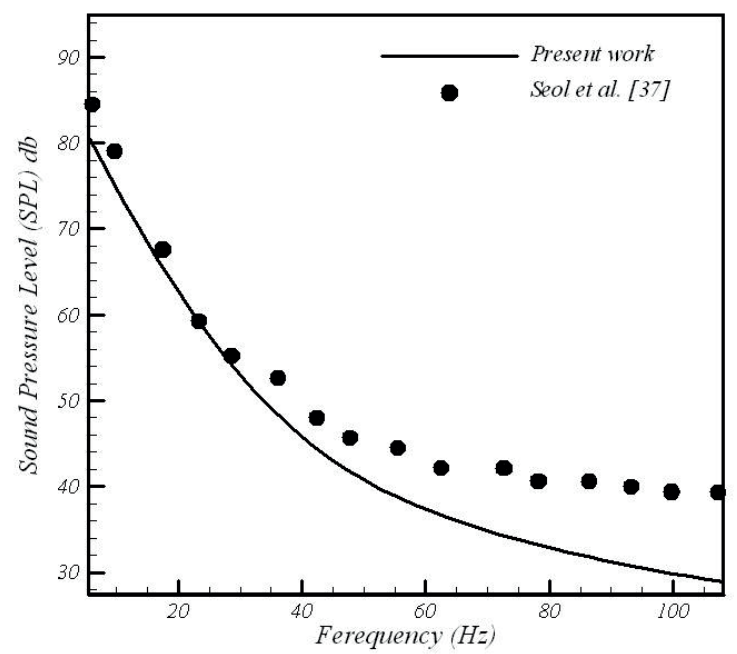

Fig. 9. Noise spectrum in receiver B 
Time histories of the flow noise for different advance coefficients were studied as well. Figure 11 shows the time dependent values of the acoustic pressure, for two advance coefficients of $0.6 \& 1$, in the receiver B. The graph evidently indicates that the values of the acoustic pressure have reached the quasi-periodic state, thus approving the convergence of the solutions in the unsteady flow. The graph also reveals that increasing the advance coefficient (decreasing the rotational speed of the propeller), leads to the decrease of the pressure amplitude. The pressure amplitude for the case with $J=0.6$ is 3.5 times larger than that for $\mathrm{J}=1.0$.

Figure 12 shows the noise spectra for different advance coefficients in hydrophone B (Figure 2). For more clear presentation, the noise spectra for the optimal $\mathrm{J}$ and lower speeds are depicted in the left figure, while the high speed cases are presented on the right. What is prominent in this figure is that the level of noise intensity per advance coefficients reaches maximum for a given frequency. This frequency is referred to as BPF (Blade Passing Frequency) and is derived from the following equation:

$$
B P F=N \times \frac{\omega}{2 \pi}
$$

In the above equation $\mathrm{N}$ is the number of propeller blades and is the angular velocity of the propeller, in radians per second. An anti-aliasing filter was used in these calculations.

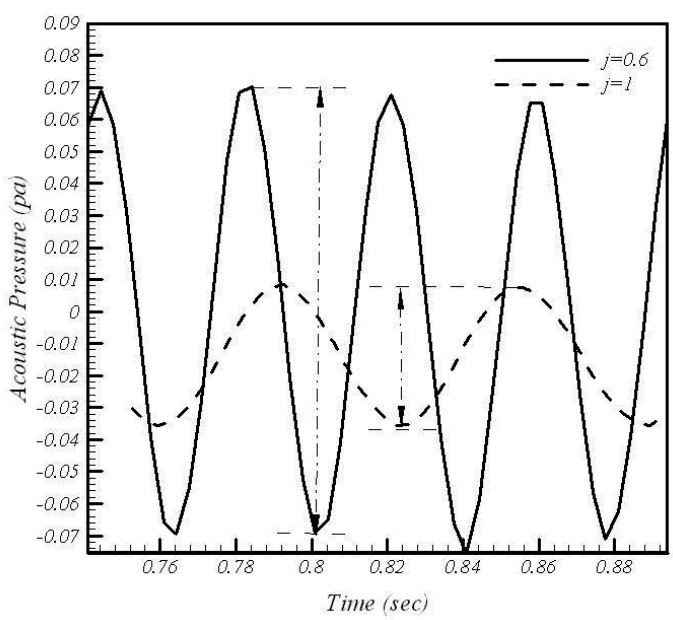

Fig.11. Time history of acoustic pressure wave for two advance coefficients
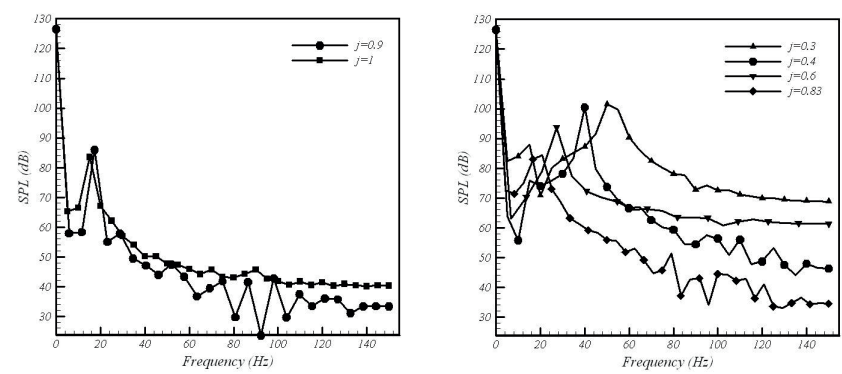

Fig. 12. Noise spectrum for different advance coefficients

\section{DIRECTIONAL DISTRIBUTION}

In the previous Section all investigations were dedicated to two locations: A and B (Figure 2). In this section, the reported results refer to all directions around the propeller in order to understand better the directional behaviour of the flow noise. For this purpose an array of 100 microphones was arranged on a sphere. The propeller was located in the centre of the sphere with the microphones and the sphere radius was $10 \mathrm{R}$.

Figure 13 shows the $3 \mathrm{D}$ graph of the intensity level of the noise recorded by 100 receivers for the frequency equal to 2 $\mathrm{Hz}$ and the rotational speed equal to $120 \mathrm{rpm}$. It is clear from Figure 13 that the maximum of the acoustic pressure is detected in the horizontal plane and the minimum of SPL is around two poles of the sphere. Although the reported results have been obtained for one special case of $120 \mathrm{rpm}$ and $2 \mathrm{~Hz}$, they clarify the directional distribution of the propeller flow noise.

Figure 14 shows the directional distribution of noise intensity in an hour circle of the sphere containing the hydrophone array. In this figure, directional distributions for two advance coefficients of $0.3 \& 0.4$ are shown. According to the figure, lower advance coefficients (higher propeller speed) result in a higher level of noise in all directions. The directional distribution reveals that the highest levels of noise are upon the hub axis, while the lowest levels are on the plane perpendicular to the propeller axis. According to the report by Seol et al., the loading noise upon the hub axis and the thickness noise on the axis perpendicular to the hub axis (the rotating plane of the propeller) are the maximal ones. Thus, it is noteworthy that, due to drastic changes of the static pressure upon the levels of the propeller in conditions of high rotational speed and low advance coefficients, the noise mostly is of loading nature.

For high advance coefficients, the directional distribution process of the hydrodynamic noise is entirely reversed (Figure 15). It is observed that the lowest levels of noise intensity are located upon the propeller hub axis and the highest levels are placed on the axis perpendicular to the hub axis (the rotating plane of the propeller). This suggests that, in the conditions of high advance coefficients (low propeller speed), the hydrodynamic noise of the propeller is of monopole type (thickness noise), as the effect of the static pressure is lower than in the case of low rotations and most of the generated noise is caused by the periodic movement and rotation of the propeller, which produces the monopole noise.

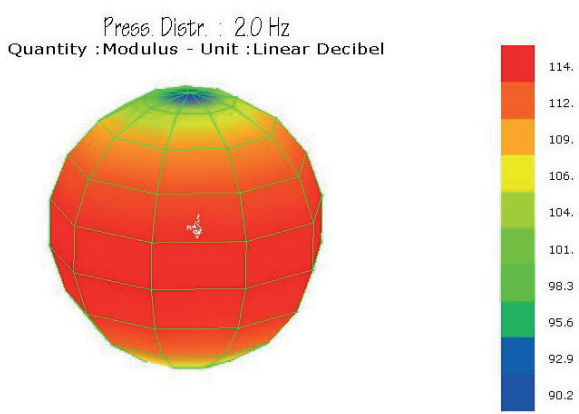

Fig. 13. SPL ( $2 \mathrm{~Hz}, 120 \mathrm{rpm})$ on the sphere around the propeller Noise spectrum for different advance coefficients 


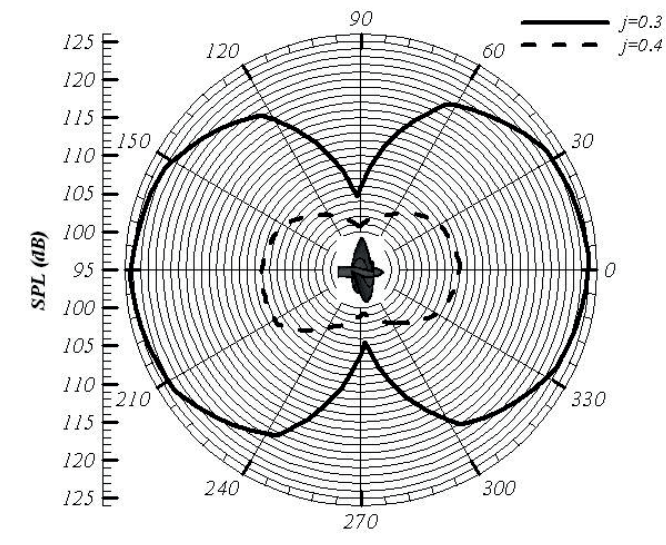

Fig. 14. Directional distribution of flow noise for low advance coefficients

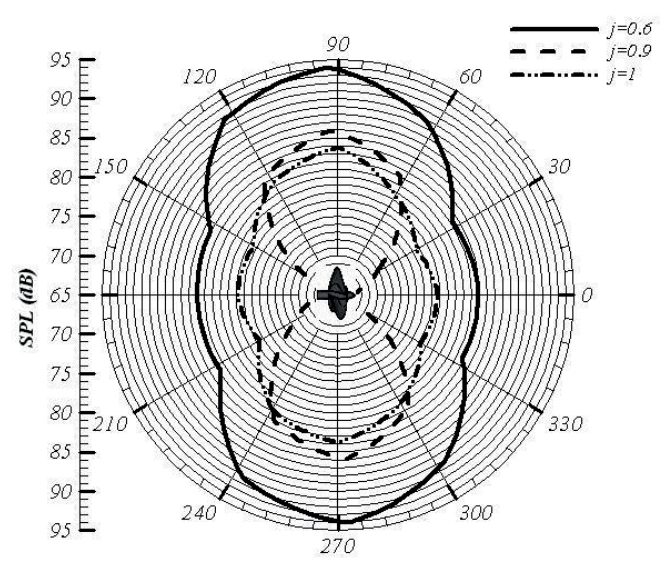

Fig. 15. Directional distribution of flow noise for high advance coefficients

\section{Broad Band Noise of the Propeller}

Another fruitful study in the area of flow noise is broadband noise investigation. In the broadband noise study regardless of the frequency, the total flow noise could be obtained. Figure 16 presents the decibel based broad band noise upon the propeller for different advance ratios. As is evident from the acoustic power distribution shown in Figure 16, the maximum sources of the generated noise are situated upon the leading edges, the blade-hub junction, and the hub tip. This is due to vortex shedding from the propeller and hub tip. We can notice that in the regions of drastic geometrical changes, a great amount of vorticity is generated (Figure 8) and subsequently large static pressure differences are observed. Thus, the maximal level of the dipole (loading) noise is produced. It is clear from the figure that as the advance ratio is increased (low speed cases), the level of noise is decreased. At J $=0.4$ the propeller hub is a source of noise while at $J=1.0$ the noise is only generated by the blades.
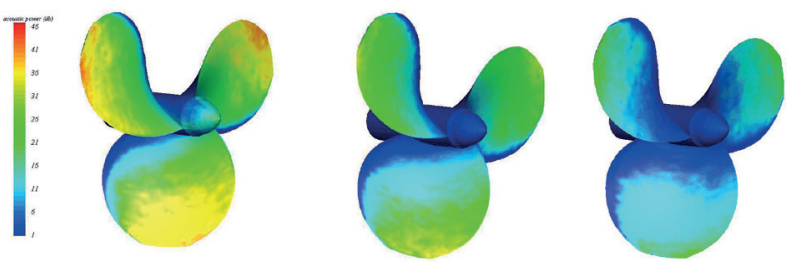

Fig. 16. Acoustic power distribution on propeller surface for $\mathrm{J}=0.4$ (left), J = 0.6 (middle) and J = 1.0 (right)

\section{CONCLUSIONS}

3D numerical simulation of the hydro-acoustic flow around a propeller has been performed and the following results have been concluded:

With the aid of the LES model of turbulence, a fairly fine grid, and a sliding mesh, the hydrodynamic flow pattern can be captured and the fluctuating quantities can be derived for further use in acoustic models.

The obtained hydrodynamic parameters have been compared against the experimental data for various advance coefficients. The observed level of agreement is satisfactory. The distributions of flow parameters such as velocity and vorticity are presented to understand better the flow.

The tonal noise of the propeller was simulated using the FWH model and the results have been compared with other available numerical results.

The effect of advance ratio has been studied and the advance ratio has been found to be a key parameter in noise propagation. Investigating the hydro-acoustic findings of the flow around the propeller has lead to the conclusion that decreasing the advance coefficients (increasing the propeller speed) increases the acoustic pressure range of the noise recorded in the receiver and decreases the period of the acoustic wave pressure.

Looking into the frequency based SPL graphs for different advance coefficients indicates that each graph reaches the maximum at a given frequency. This frequency is referred to as BPF (Blade Passing Frequency).

The directional distribution of the noise power level around the propeller should also be taken into consideration. In low advance coefficients (high speeds), the level of the noise around the hub axis of the propeller increases to the maximum, and drops to the minimum upon the axis perpendicular to the propeller axis within the rotating plane of the propeller. This is an indication of dipole (loading) noise maximisation in the examined cases. However, in high advance coefficients (low speed), the noise power within the rotating plane of the propeller increases to the maximum, and drops to the minimum upon the axis of the propeller hub. This is an 
indication of the monopole (thickness) noise capability in these advance coefficients.

A broadband study has been carried out on the propeller, and the regions with highest noise potential have been detected, see the $3 \mathrm{D}$ graph. It is noticeable that the flow pattern, especially the vorticity distribution is associated with the broadband noise.

Examination of the broad band noise upon the propeller surface and the resultant graph of the acoustic power plainly demonstrate that regions in the vicinity of propeller hub and tip play a crucial role in the generation of vortices in the flow around the propeller, which are precisely the source of major part of the noise generated around the propeller. In these regions, due to drastic geometrical changes, high static pressure differences are observed.

\section{BIBLIOGRAPHY}

1. Amini H., Steen S.: Experimental and Theoretical Analysis of Propeller Shaft Loads in Oblique Inflow, Journal of Ship Research, Vol. 55 No 4, pp. 268-288(21), 2011.

2. Juan J.A., Kazuo N., Claudio M.S., Julio C.A.: Experimental Investigation of the Hydrodynamic Coefficients of a Remotely Operated Vehicle Using a Planar Motion Mechanism, Journal Offshore Mech. Arct. Eng, Vol. 134, Issue 2, 021601 (6 pages), 2012.

3. Ning L., Sande W., Tao G., Xiyou L., Ziyang Y.: Experimental research on the double-peak characteristic of underwater radiated noise in the near field on top of a submarine, Journal of Marine Science and Application, Vol. 10 N0 2, 233-239, DOI: 10.1007/s11804-011-1049-2

4. Zhi-hua L, Ying X, Zhan-zhi W, Song W, Cheng x.: Numerical Simulation and Experimental Study of the new Method of Horseshoe Vortex Control, Journal of Hydrodynamics, Ser. B, Vol. 22, Issue 4, pp. 572-581, 2010.

5. Gaggero S., Villa D., Brizzolara S.: RANS and PANEL method for unsteady flow propeller analysis, Journal of Hydrodynamics, Vol. 22, Issue 5, Supplement 1, pp. 564-569, 2010.

6. Fang-wen H., Shi-tang D.: Numerical Analysis for Circulation Distribution of Propeller Blade, Journal of Hydrodynamics, Vol. 22, Issue 4, pp. 488-493, 2010.

7. Ghasseni H., Ghadimi P.: Numerical analysis of the high skew propeller of an underwater vehicle, Journal of Marine Science and Application Vol. 10 No. 3, 289299, DOI: 10.1007/s11804-011-1071-4

8. Baltazar J., Falcão de Campos J.A.C.: An iteratively coupled solution of the cavitating flow on marine propellers using BEM, Journal of Hydrodynamics, Ser. B, Vol. 22, Issue 5, Supplement 1, pp. 838-843, 2010.

9. G. Kuiper.: New developments and propeller design, Journal of Hydrodynamics, Vol. 22, Issue 5, Supplement 1, pp. 7-16, 2010.
10. Bong-Jun C., Seokcheon G.: Study on a procedure for propulsive performance prediction for CRP-POD systems, Journal of Marine Science and Technology, Vol. 16 No. 1, pp.1-7, DOI: 10.1007/s00773-010-0108-8

11. Fang-wen H., Shi-tang D.: Numerical simulation of the structure of propeller's tip vortex and wake, Journal of Hydrodynamics, Vol. 22, Issue 5, Supplement 1, pp. 457-461, 2010.

12. Chao W., Sheng H., Xin C., Miao H.: Applying periodic boundary conditions to predict open water propeller performance, Journal of Marine Science and Application, Vol. 9 No. 3, 262-267, DOI: 10.1007/s11804-010-1005-6

13. Chun-yu G., Wen-ting H., Sheng H.: Using RANS to simulate the interaction and overall performance of propellers and rudders with thrust fins, Journal of Marine Science and Application, Vol. 9 No. 3, 323-327, DOI: 10.1007/s11804-010-1015-4

14. Wilson P.A., Molland, A.F., The development of hydrodynamics: 1860-2010. In, The William Froude Conference: Advances in Theoretical and Applied Hydrodynamics, Past and Future, Portsmouth, UK, $24-25,2010$.

15. Liu Z., Xiong Y., Tu C.: Numerical simulation and control of horseshoe vortex around an appendage-body junction, Journal of Fluids and Structures, Vol. 27, Issue 1, pp 23-42, 2011.

16. Alin N., Bensow R.E., Fureby C.; Huuva T., Svennberg U.: Current Capabilities of DES and LES for Submarines at Straight Course, Journal of Ship Research, Vol. 54, Number 3, pp. 184-196(13), 2010.

17. Jong-Yeon H., Kyung-Soo Y., Klaus B.: Direct Numerical Simulation of Turbulent Flow Around a Rotating Circular Cylinder, J. Fluids Eng, Vol. 129, Issue 1, 40-47, doi:10.1115/1.2375133, 2007.

18. Wilcox D. C., Turbulence Modeling for CFD, 3rd edition, DCW Industries, Canada, 2006.

19. Yu-cun P., Huai-xin Z.: Numerical hydro-acoustic prediction of marine propeller noise, Journal of Shanghai Jiaotong University (Science), Vol. 15 No. 6, 707-712, DOI: $10.1007 /$ s12204-010-1073-4

20. Rickard E., B Bark., G Bark.: Implicit LES Predictions of the Cavitating Flow on a Propeller, Journal Fluids Eng, Vol. 132, Issue 4, 041302 (10 pages) doi:10.1115/1.4001342, 2010.

21. Nai-xian L., Rickard E.B., Bark G.: LES of unsteady cavitation on the delft twisted foil, Journal of Hydrodynamics, Ser. B, Vol. 22, Issue 5, Supplement 1, pp. 784-791, 2010.

22. C Fureby.: ILES and LES of Complex Engineering Turbulent Flows, Journal Fluids Eng, Vol. 129, Issue 12, 1514 (10 pages) doi:10.1115/1.2801370, 2007.

23. Oberai A., Ronaldkin F., Hughes T.: Computational procedures for determining structural-acoustic response due to hydrodynamic sources, Comp. Methods Appl. Mech. Engineering Vol. 190, pp. 345-361, 2000.

24. Oberai A., Roknaldin F., Hughes T.J.R.: Computation of Trailing-Edge Noise due to Turbulent Flow over an Airfoil, AIAA Aeroacoustics Journal Vol. 40, pp.2206-2216, 2002. 
25. Caro S., Ploumhans P., Gallez X.: A new formulation based on Lighthill's Analogy applied to an idealized Automotive HVAC Blower using AcuSolve and Actran/ $L A$, Proceedings of 11th AIAA/CEAS Aeroacoustics Conference, No. 3015, Monterey, USA, 2005.

26. Damiano C.: Aeroacoustics research in Europe: The CEAS-ASC report on 2009 highlights, Journal of Sound and Vibration, Vol. 329, Issue 22, 25, pp. 4810-4828, 2010.

27. Yu-cun P., Huai-xin Z.: Numerical hydro-acoustic prediction of marine propeller noise, Journal of Shanghai Jiaotong University (Science), Vol. 15 No. 6, 707-712, DOI: $10.1007 / \mathrm{s} 12204-010-1073-4$

28. Ruchonnet N., Alligné S., Nicolet C., Avellan F.: Cavitation influence on hydroacoustic resonance in pipe, Journal of Fluids and Structures, 10.001, 2011.

29. Young-Zehr K., Jui-Hsiang K,: Underwater acoustic field and pressure fluctuation on ship hull due to unsteady propeller sheet cavitation, Journal of Marine Science and Technology, Vol. 16 No. 3, pp. 241-253, DOI: 10.1007/ s00773-011-0131-4, 2011

30. Bogey C., Bailly C., Juv'e D.,: Computation of flow noise using source terms in linearized Euler's equations, AIAA Journal Vol. 40 No. 2, pp. 235-243, 2002.

31. Utzmann J., Schwartzkopff T., Dumbser M., Munz C.D.: Heterogeneous Domain Decomposition for CAA, Proceedings of 11th AIAA/CEAS Aeroacoustics Conference, Monterey, USA, 2005.

32. Zhao Y., J. Morris P., The Prediction of Fan Exhaust Noise Propagation, 11th AIAA Aeroacoustics Conference, No. 2005-2815, Monterey, USA, 2005.

33. Ewert R., Schröder W.,: Acoustic Perturbation Equations based on Flow decomposition via Source Filtering, Journal of Computational Physics Vol. 188, pp. 365-398, 2003.
34. Seo J., Moon Y., Linearized perturbed compressible equations for low Mach number aeroacoustics, Journal of Computational Physics Vol. 218 No. 2, pp 702-719, 2006.

35. Ffowcs-Williams J. E., Hawkings D.L.: Sound Generation by Turbulence and Surfaces in Arbitrary Motion, Proc. Roy. Soc. London, A264:321-342, 1969.

36. Proudman I.: The Generation of Noise by Isotropic Turbulence, Proc. Roy. Soc., A214:119, 1952.

37. Y. Pan, H. Zhang,: Numerical Hydro-Acoustic Prediction of Marine Propeller Noise, Journal of Shanghai Jiaotong Univ 15(6), pp. 707-712, 2010

38. Seol H., Such J-C., Lee S.,: Development Of Hybrid Method for Prediction of Underwater Propeller Noise, Journal of Sound and Vibration.Vol..288, pp. 345-360, 2005.

\section{CONTACT WITH THE AUTORS}

S. Kheradmand
A. Rahrovi
B. Mousavi

Department of Mechanical and Aerospace Engineering Malek-Ashtar University of Technology

Shahin-shahr

P.O. Box $83145 / 115$

Isfahan

Iran

kheradmand@mut-es.ac.ir 